\title{
Large Deflection Analysis of Inelastic Plane Frames
}

\author{
By T. Y. Kam ${ }^{1}$
}

\begin{abstract}
Agstract: A general method for the large deflection analysis of inelastic plane frames is presented. The large deflection analysis is based on the Eulerian formulation in which a member tangent stiffness matrix is constructed with reference to the current deformed configuration. The exact differential equation and the moment-thrust-curvature relations of a member subjected to large relative deflection are used for deriving the member tangent stiffness matrix. A member tangent stiffness matrix for $W$-sections of elasto-plastic material is constructed as an example and its application to the large deflection analysis of inelastic frames is demonstrated by several examples. A simple form of the member tangent stiffness matrix is deduced from the above exact formulation by utilizing the assumption of small member relative deflections. It is shown that the simple one can yield excellent results compared to those obtained by the exact formulation. Hence it is reasonable to adopt the assumption of small member relative deflections in the large deflection analysis of inelastic framed structures.
\end{abstract}

\section{INTRODUCTION}

The evaluation of accurate ultimate loads of structures is an important task in the structural design and reliability analysis processes. In order to find the accurate ultimate loads, one has to consider both material and geometrical nonlinearities in the structural analysis. Therefore the nonlinear analysis of framed structures has become a subject of research for many years. A considerable amount of research was devoted to the analysis of inelastic frames based on the assumption of small deflections of the frames (Alvarez and Birnstiel 1969; Harung and Miller 1973; Kam et al. 1983; Oran 1973; Saafan 1963; Vijakklane 1974). Some researchers investigated the large deflections of elastic frames (Lee et al. 1968; Meek and Tan 1984; Oran and Kassimali 1976; Qashu and Dadeppo 1983; Turner et al. 1960). Recently, the large deflection analysis of inelastic frames has been successfully treated by some authors (Argyris et al. 1982; Backlund 1976; Bathe and Ozdemir 1975; Cichon 1984). For instance, Kassimali (1983) presented a method for the analysis of inelastic frames in which rigid body displacements of members can be arbitrarily large while relative member deflections are considered to be small and yielding is restricted to concentrated points at member ends. El-Zanaty and Murray (1983) utilized the incremental viriational principles together with the finite element method to analyze inelastic frames subjected to large deflections. Nevertheless it has been pointed out that the development of an efficient and effective method for the large deflection analysis of inelastic structures is

'Prof. Mech. Engrg. Dept., National Chiao Tung Univ., Hsinchu, Taiwan, R.O.C.

Note. Discussion open until June 1, 1988. To extend the closing date one month, a written request must be filed with the ASCE Manager of Journals. The manuscript for this paper was submitted for review and possible publication on September 10, 1986. This paper is part of the Journal of Structural Engineering, Vol. 114, No. 1, January, 1988. (C) ASCE, ISSN 0733-9445/88/0001-0184/\$1.00+\$.15 per page. Paper No. 22139. 
still a live problem (Cichon 1984). Hence more research work is needed in this area.

In this paper, an alternative for the large defiection analysis of inelastic frames is proposed. A general formulation of member tangent stiffness matrix for inelastic members subjected to large deffection is presented. The formulation is derived from an integral equation form of the exact differential equation of a beam-column in a local Eulerian coordinate system and includes the effects of the spreading of yielding, changes in the stiffness of the structure due to axial force, axial shortening of members, and the reduction of plastic moments of resistance due to axial loads. The tangent stiffness matrix of a wide-flange steel member is presented as an example, and its application to the large deflection analysis of inelastic frames is demonstrated by means of several examples. A simpler form of the member tangent stiffness matrix obtained by adopting the assumption of small relative member deflections is used for the large deflection analysis of the previously mentioned inelastic frames to study the effect of different formulations of member tangent stiffness on the behavior of the frames. It is shown that for some inelastic frames the effect is small when the large displacements of the frames are mainly due to the rigid body displacements of members and member relative deffections remain small during deformation.

The basic assumptions adopted in the formulation of the member tangential stiffness matrix are: the member is initially straight; and sections normal to the centroidal axis of the undeformed member remain undistorted and normal to the deformed centroidal axis.

\section{Large Deflection of Member at Equilibrium State}

Consider an arbitrary prismatic member $i j$ of initial length $L_{0}$ in equilibrium under the action of end forces in local Eulerian coordinates as shown in Fig. 1 in which the positive directions of the end forces and end displacements are indicated.

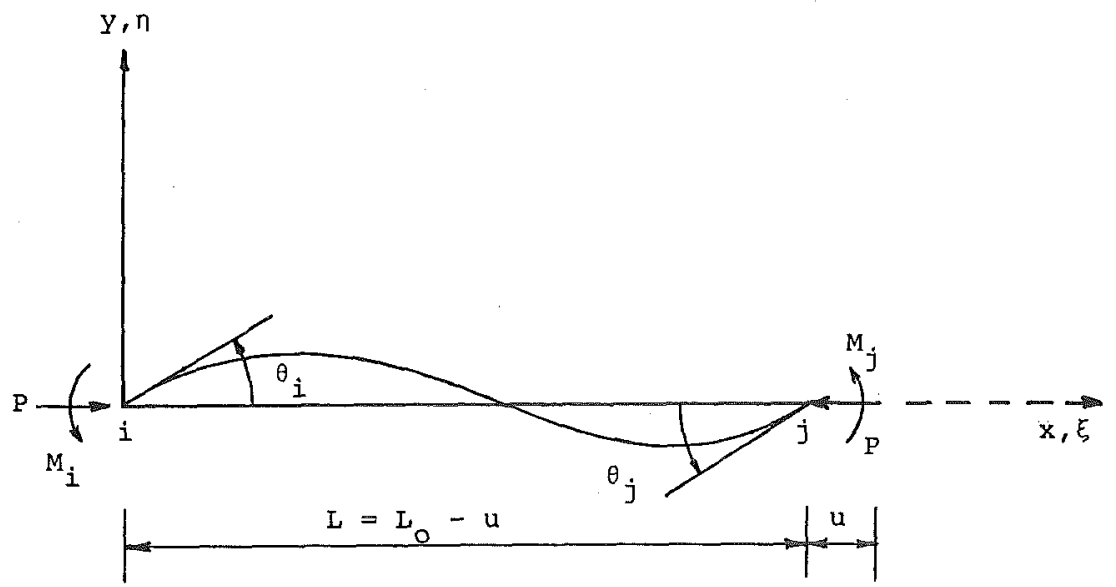

FIG. 1. Member in Local Eulerian Coordinate System 


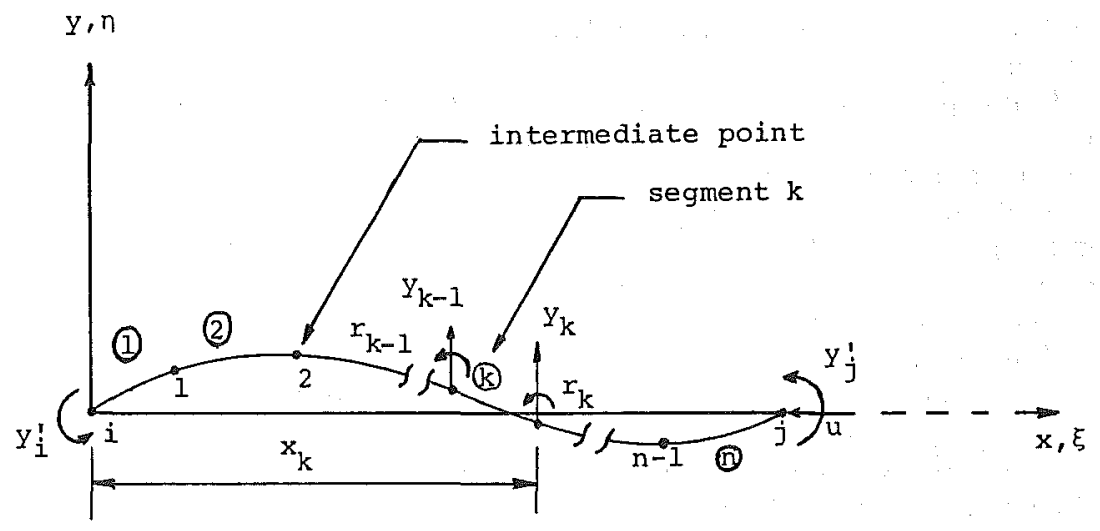

FIG. 2. Deflections at Intermediate Points

The differential equation of the member with large relative deflection is $y^{\prime \prime}=\alpha \Phi(a)$

in which $\alpha=\left[1+\left(y^{\prime}\right)^{2}\right]^{3 / 2} ; \Phi(a)=$ the curvature at any section of the member; and $a$ is a set of independent parameters. Integrating Eq. 1 twice and observing the boundary conditions at the two ends of the member, i.e., $y=0$ at $x=0$ and $x=L$, one obtains the following equations:

$y^{\prime}=\int_{0}^{x} \frac{\xi \alpha \Phi}{L} d \xi-\int_{x}^{L}\left(1-\frac{\xi}{L}\right) \alpha \Phi d \xi$

and

$y=-\int_{0}^{x}\left(1-\frac{x}{L}\right) \xi \alpha \Phi d \xi-\int_{x}^{L} x\left(1-\frac{\xi}{L}\right) \alpha \Phi d \xi$.

where $\xi$ is a dummy variable for integration. The slopes at the two ends can be written as

$y_{i}^{\prime}=-\int_{0}^{L}\left(1-\frac{\xi}{L}\right) \alpha \Phi d \xi$

$y_{j}^{\prime}=\int_{0}^{L} \frac{\xi}{L} \alpha \Phi d \xi$

Subtraction of $y_{i}^{\prime}$ from $y_{j}^{\prime}$ gives

$y_{j}^{\prime}-y_{i}^{\prime}=\int_{0}^{L} \alpha \Phi d \xi$

If member $i j$ of Fig. 1 is subdivided into $n$ segments with $n-1$ intermediate points between them, as shown in Fig. 2, the vertical deflections and the 
slopes at the intermediate points from Eq. 2 are

$y_{k}=-\int_{0}^{x_{k}}\left(1-\frac{x_{k}}{L}\right) \xi \alpha \Phi d \xi-\int_{x_{k}}^{L} x_{k}\left(1-\frac{\xi}{L}\right) \alpha \bar{\Phi} d \xi$

and

$r_{k}=\int_{0}^{x_{k}} \frac{\xi \alpha \Phi}{L} d \xi-\int_{x_{k}}^{L}\left(1-\frac{\xi}{L}\right) \alpha \Phi d \xi$

in which $y_{k}, r_{k}=$ deflections and slopes at the intermediate points, respectively; and $k=1,2, \ldots, n-1$. The axial deformation of member $i j$ due to the application of axial forces and the bowing effect is expressed as

$u=\int_{0}^{L} \varepsilon_{c} d \xi+\int_{0}^{L} \sqrt{1+\left(y^{\prime}\right)^{2}} d \xi-L$

in which $u=$ shortening in axial direction; and $\varepsilon_{c}=$ compressive normal strain at the centroid of the cross section in the deformed state. Let the displacement relations of Eqs. 6, 4, 3b, and 5 be expressed in the functional forms

$F_{1}(a)=\int_{0}^{L} \varepsilon_{c} d \xi+\int_{0}^{L} \sqrt{1+\left(y^{\prime}\right)^{2}} d \xi-L-u$

$F_{2}(a)=\int_{0}^{L} \alpha \Phi d \xi-\left(y_{j}^{\prime}-y_{i}^{\prime}\right)$

$F_{3}(a)=\int_{0}^{L} \frac{\xi \alpha \Phi}{L} d \xi-y_{j}^{\prime}$

$H_{k}(a)=-\int_{0}^{x_{k}} \xi\left(1-\frac{x_{k}}{L}\right) \alpha \Phi d \xi-\int_{x_{k}}^{L} x_{k}\left(1-\frac{\xi}{L}\right) \alpha \Phi d \xi-y_{k}$

$K_{k}(a)=\int_{0}^{x_{k}} \frac{\xi \alpha \Phi}{L} d \xi-\int_{x_{k}}^{L}\left(1-\frac{\xi}{L}\right) \alpha \Phi d \xi-r_{k}$

in which $k=1,2, \ldots, n-1$; and $a$ is defined as

$a=\left[\begin{array}{l}P M_{i} M_{j} y_{1} \ldots y_{n-1} r_{1} \ldots r_{n-1} u y_{i}^{\prime} y_{j}^{\prime}\end{array}\right]$

Define a column vector $F$ such that

$\mathbf{F}(a)^{T}=\left[F_{1} F_{2} F_{3} H_{1} \ldots H_{n-1} K_{1} \ldots K_{n-1}\right]$

When compatibility and equilibrium are satisfied, $\mathbf{F}$ becomes a null vector, or

$\mathbf{F}(a)=0$

The evaluation of the integrals in Eq. 7 can be accomplished by using cubic splines to approximate the deflections of the segments along the member and a Gauss-Lobatto quadrature expression for numerical integration. The use of a Gauss-Lobatto quadrature, which considers the end points as integration points, can ensure convergence and give excellent results for 
the plastic analysis of members with plastic hinges developed at the ends as reported in Crisfield (1983).

If the end displacements of member $i j$ are prescribed, the corresponding end forces and deflections at the intermediate points of the member can be evaluated from Eq. $8 b$ by using the standard Newton-Raphson iteration procedure. Let

$a=\left[a_{1} \vdots \vdots a_{2}\right]$

in which

$a_{1}=\left[P M_{i} M_{j} y_{1} \ldots y_{n-1} r_{1} \ldots r_{n-1}\right]$

and

$a_{2}=\left[u y_{i}^{\prime} y_{j}^{\prime}\right]$

Assuming $\mathbf{F}(a)$ to be analytic, and since $a_{2}$ is prescribed, expansion of $\mathbf{F}(a)$ in a truncated Taylor series about $a_{1}^{n}$ which is an approximation of $a_{1}$ yields

$F\left(\left[a_{1}^{n+1} \vdots a_{2}\right]\right)=F\left(\left[a_{1}^{n} \vdots a_{2}\right]\right)+\left[\frac{d F^{n}}{d a_{1}}\right]\left(\Delta a_{1}^{t}\right)^{n} \cong 0$

in which $a_{1}^{n+1}=a_{1}^{n}+\Delta a_{1}^{n} ; a^{t}=$ transpose of $a$; and $n$ stands for the $n$th iteration cycle of the iteration procedure. Improved values of $a_{1}^{n+1}$ can then be obtained by computing

$\Delta a_{1}^{n}=-\left[\frac{d F^{n}}{d a_{1}}\right]^{-1} F^{n}$

Repetition of the process continues until the error

$e=F^{n+1}-F^{n}$

becomes sufficiently small.

\section{Member Tangential Stiffness Matrix in Local. Eulerian Coordinates}

Consider an adjacent equilibrium state which is obtained by incrementing the end displacements and end forces of the member which is currently in equilibrium. Then Eq. 8 becomes

$F(a+\Delta a)=0$

Expansion of Eq. 13 in a curtailed Taylor series leads to

$F(a+\Delta a)=F(a)+\left[\frac{d F}{d a}\right] \Delta a^{t}=0$

and since $F(a)=0$

$\left[\frac{d F}{d a}\right] \Delta a^{t}=0$ 
From Eq. 9, Eq. 15 can be written as

$\left[\frac{d F}{d a_{1}}\right] \Delta a_{1}^{t}=-\left[\frac{d F}{d a_{2}}\right] \Delta a_{2}^{t}$

If

$b=\left[P M_{i} M_{j}\right]^{t}$

and

$q=\left[\begin{array}{llllll}y_{1} & \ldots & y_{n-1} r_{1} & \ldots & r_{n-1}\end{array}\right]^{t}$

then

$a_{1}=\left[b^{t} \vdots q^{t}\right]$

Substitution of Eq. 18 into Eq. 16 yields

$\left[\frac{d F}{d a_{1}}\right]\left\{\begin{array}{l}\Delta b \\ \dddot{\Delta q}\end{array}\right\}=-\left[\frac{d F}{d a_{2}}\right] \Delta a_{2}^{t}$

Performing a static condensation of Eq. 19, a relationship between $\Delta b$ and $\Delta a_{2}$ is obtained as

$\Delta b=K^{\prime} \Delta a_{2} t$

in which $K^{\prime}=a 3 \times 3$ member tangential stiffness matrix in local Eulerian coordinates.

\section{Transformation of Coordinates}

Fig. 3 shows the initial undeformed and the current deformed configurations of member $i j$. The rigid body rotation of the member can be

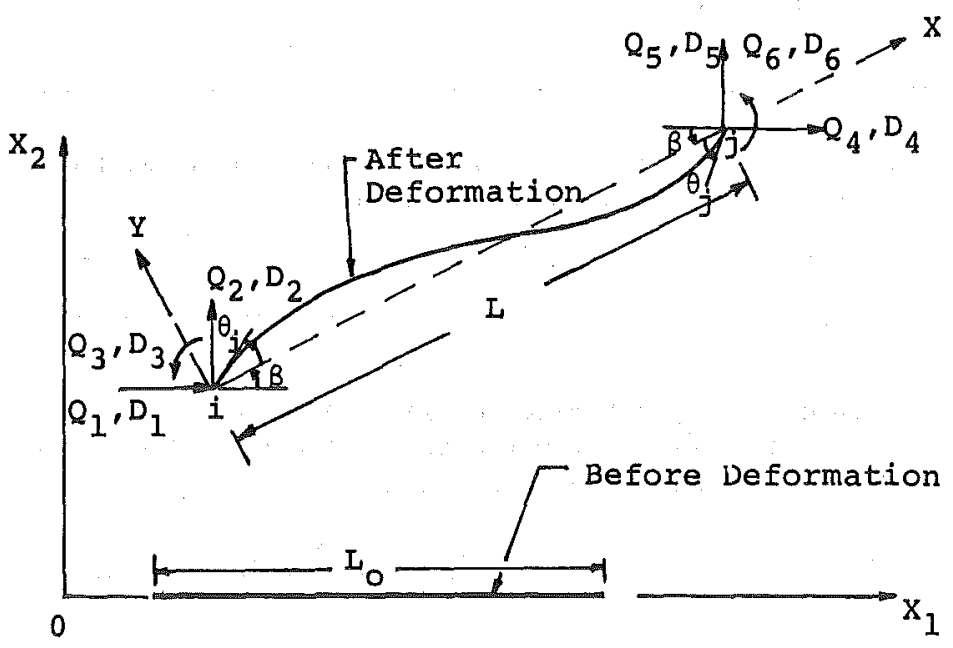

FIG. 3. Member in Global Coordinate System 
arbitrarily large. Let $Q$ and $D$ be the member end forces and end displacements, respectively, of the deformed member in the global $x_{1}-x_{2}$ coordinate system. The incremental global member end forces $\Delta Q$ are related to those in the local Eulerian coordinate system, i.e., $\Delta b$, by

$\Delta Q=T \Delta b$

in which

$\Delta Q^{t}=\left[\Delta Q_{1} \Delta Q_{2} \Delta Q_{3} \Delta Q_{4} \Delta Q_{5} \Delta Q_{6}\right]$

and the transformation matrix

$\mathbf{T}=\left[\begin{array}{ccc}\cos \beta & -\frac{\sin \beta}{L} & -\frac{\sin \beta}{L} \\ \sin \beta & \frac{\cos \beta}{L} & \frac{\cos \beta}{L} \\ 0 & 1 & 0 \\ -\cos \beta & \frac{\sin \beta}{L} & \frac{\sin \beta}{L} \\ -\sin \beta & \frac{\cos \beta}{L} & \frac{\cos \beta}{L} \\ 0 & 0 & 1\end{array}\right]$

in which $\beta=$ angle between the local and global coordinate axes. The slope at any section of the member referenced to the local Eulerian coordinate system is

$y^{\prime}=\tan \theta$

in which $\theta=$ rotation of the section in the local coordinate system, and the incremental relation between slope and rotation is

$\Delta y^{\prime}=\frac{1}{\cos ^{2} \theta} \Delta \theta$

In view of Eq. 23 the incremental relation between member end displacements $\Delta D$ in the global coordinate system and those, $\Delta a_{2}$, in the local coordinate system can be written as

$\Delta a_{2}=B^{t} \Delta D$

in which

$\Delta D^{t}=\left\{\Delta D_{1} \Delta D_{2} \Delta D_{3} \Delta D_{4} \Delta D_{5} \Delta D_{6}\right\}$ 
and the transformation matrix

$$
\mathbf{B}=\left[\begin{array}{ccc}
\cos \beta & -\frac{\sin \beta}{L \cos ^{2} \theta_{i}} & -\frac{\sin \beta}{L \cos ^{2} \theta_{j}} \\
\sin \beta & \frac{\cos \beta}{L \cos ^{2} \theta_{i}} & \frac{\cos \beta}{L \cos ^{2} \theta_{j}} \\
0 & \frac{1}{\cos ^{2} \theta_{i}} & 0 \\
-\cos \beta & \frac{\sin \beta}{L \cos ^{2} \theta_{i}} & \frac{\cos \beta}{L \cos ^{2} \theta_{j}} \\
-\sin \beta & -\frac{\cos \beta}{L \cos ^{2} \theta_{i}} & \frac{\cos \beta}{L \cos ^{2} \theta_{j}} \\
0 & 0 & \frac{1}{\cos ^{2} \theta_{j}}
\end{array}\right]
$$

in which $\theta_{i}, \theta_{j}=$ member end rotations in a local coordinate system. From Eqs. 21 and 24 the incremental relationship between member end forces and end displacements in the global coordinate system can be expressed as

$\Delta Q=K \Delta D$

and the $6 \times 6$ member tangential stiffness matrix

$\mathbf{K}=T K^{\prime} B^{t}$

Assembling all member tangential stiffness matrices by the direct stiffness procedure yields the structure tangential stiffness matrix for relating incremental values of nodal forces and nodal displacements as

$\Delta R=K_{s} \Delta r$

in which $K_{s}=$ the structure tangential stiffness matrix; $\Delta R=$ the assembled vector of incremental nodal forces; and $\Delta r=$ the assembled vector of incremental nodal displacements. As for the nonlinear analysis of inelastic frames, the modified arc length method of Crisfield (1983) and Meek and Tan (1984) is used for obtaining the load-displacement characteristics of the frames.

It is noted that the foregoing formulation of the member tangential stiffness matrix will become much simpler if the relative member deflection in the local coordinate system is assumed to be small while the rigid body rotation of the member can be arbitrarily large. In this occasion, the variable $\alpha$ of Eq. 1 becomes one, the slope of the member in the local coordinate system can be approximated by its rotation, and the transformation matrice $\mathbf{B}$ in $\mathrm{Eq} .25 b$ can be replaced by $\mathrm{T}$ of $\mathrm{Eq} .21 c$. This leads to a simpler form of the member tangent stiffness matrix. The use of this stiffness matrix for the large deflection analysis of the same inelastic frames can yield excellent results as shown in the subsequent sections. 


\section{Stiffness Matrix of Wide-Flange Members}

If a wide-flange member undergoes elastic-plastic bending, three possible stress distributions, which correspond, respectively, to three zoneselastic, primary plastic, and secondary plastic-may occur at a section of the member. The three stress distributions of an idealized wide-flange section are shown in Fig. 4. Explicit expressions of curvature in terms of bending moment and axial force on a cross section for the three zones have been derived by Hauck and Lee (1963) with the following assumptions: (1) The material is linear elastic-perfectly plastic; (2) cooling residual stress is not included; and (3) no strain reversal is considered. The spreading of yield zone in a member is considered by substituting the appropriate curvature expressions into Eqs. 2 and 6 when performing the integrations. The reduction of plastic moment due to axials loads is implicitly included in the curvature expressions. From these curvature equations, the tangent stiffness matrix of Eq. 20 can be constructed by following the previously described procedure. A complete description of the formulation is given in Kuo (1985).

\section{Numerical EXample}

The accuracy of the proposed method is first verified by comparing the present analysis of the post-buckling of a beam column with the exact

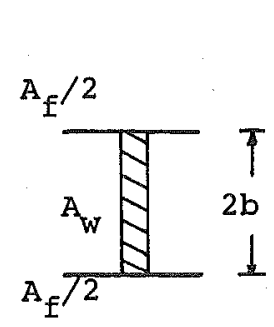

(a)

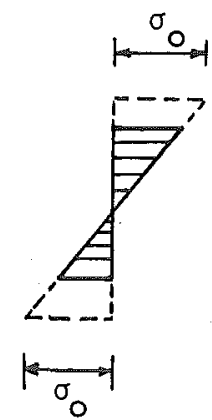

(b)

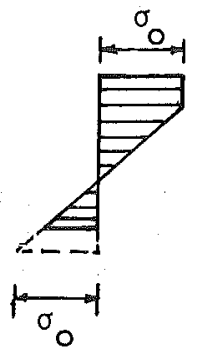

(c)

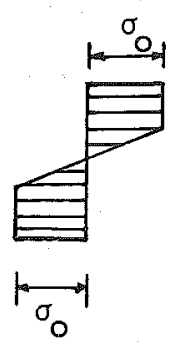

(d)

FiG. 4. Idealized Section and Types ở Stress Distribution: (a) Idealized Section; (b) Elastic; (c) Primary Plastic; (d) Secondary Plastic

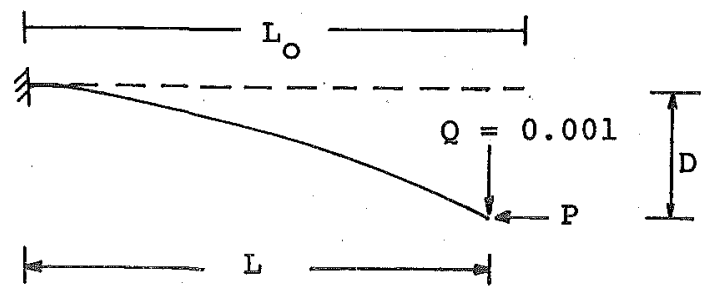

FIG. 5. Loads on Elastic Beam-Column 


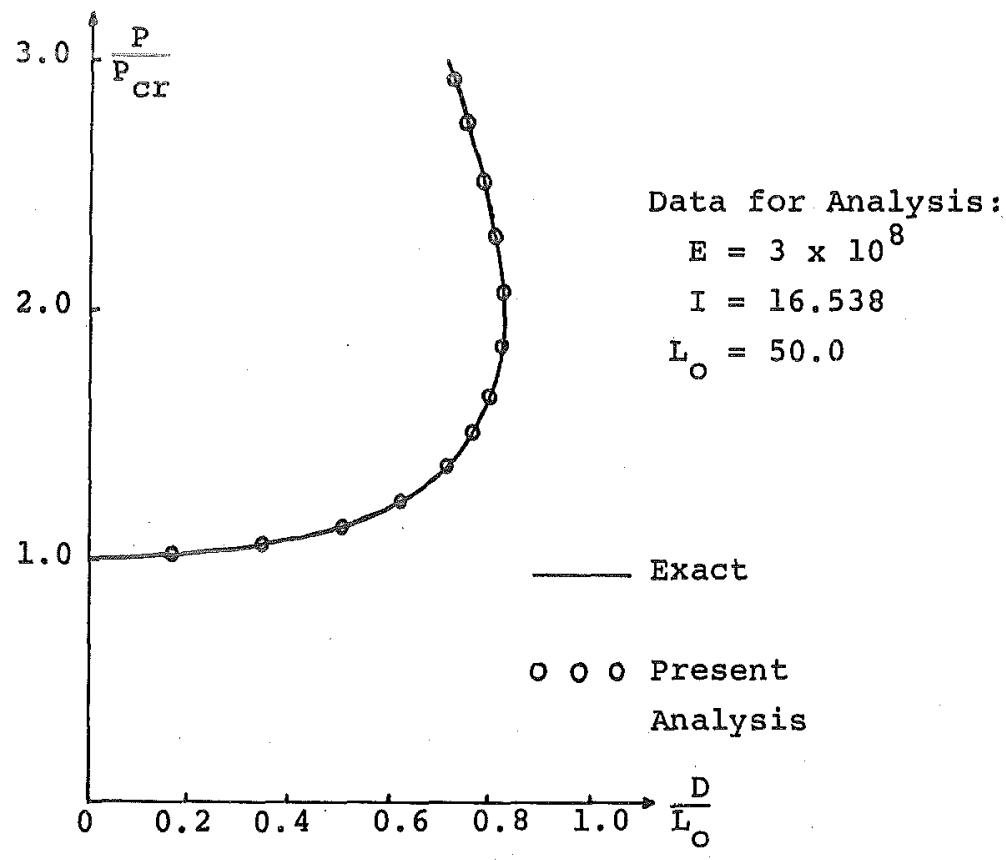

FIG. 6. Post-Buckling of Elastic Beam-Column

solution. The cantilever beam-column is subjected to an axial force $P$ as shown in Fig. 5. A small transverse load is applied to create an initial imperfection. The results on the load-displacement relation obtained by the present method are plotted in Fig. 6 and compared with the "Elastica" solution (Timoshenko and Gere 1961). The application of the proposed method in the large deflection analysis of inelastic frames is demonstrated by analyzing two frames. Both exact and approximate formulations of the member tangent stiffness matrix are used for the illustrations and the results are compared.

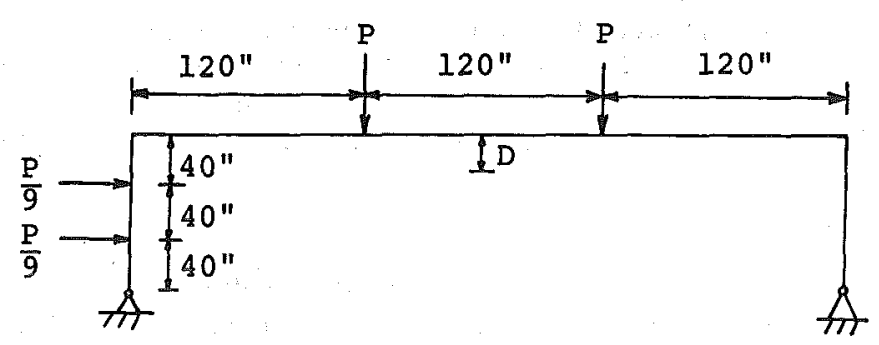

FIG. 7. Loaded Frame with Hinged Base ( $\uparrow$ in. $=25.4 \mathrm{~mm}$ ) 


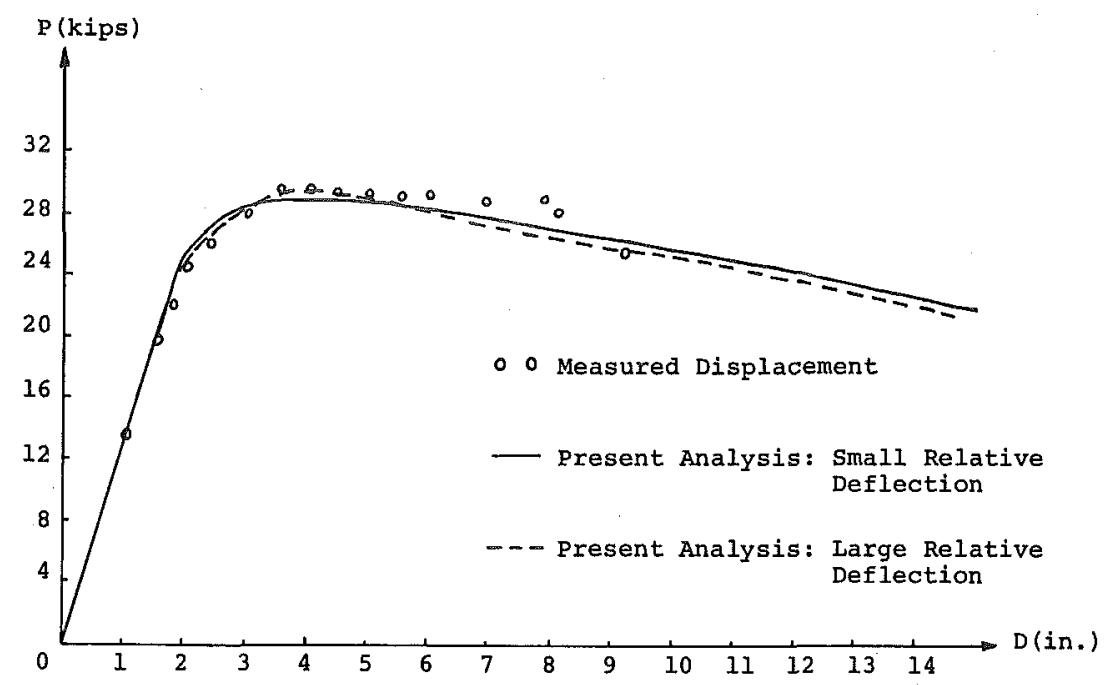

FIG. 8. Load-Displacement Curves for Frame with Hinged Base ( 1 kip $=4448 \mathrm{~N}$; 1 in. $=25.4 \mathrm{~mm}$ )

\section{Comparison with Experimental Frame}

The frame with a hinged base in Fig. 7 was fabricated from $W 12 \times 36$ steel sections and tested by Schilling et al. (1956). Loads were applied at the four points indicated and increased in proportion by small finite increments. The deflection at the midspan of the beam was measured and the resulting experimental points are shown in Fig. 8.

Using the member properties obtained from actual measurements, the frame is analyzed by using the present method in which seven members are used with each member being subdivided into six segments and a 4 point Gauss-Lobatto quadrature expression is used for numerical integration within each segment. Results obtained from the two different formulations based on large and small member deflection theories, respectively, are plotted in Fig. 8.

\section{Load-Displacement Relation of Four-Story Frame}

The four-story frame in Fig. 9 was previously analyzed for different lateral to vertical load ratios $(r=0.1,0.24,0.5)$ by Kassimali (1983) using large rigid body rotation and small relative member deflection theory, and the results are shown in Fig. 10. The present method is utilized to analyze the same frame. The results obtained from the formulations based on large and small relative member deflection theories are plotted in Fig. 10.

\section{Conclusions}

A general method for the large deflection analysis of elastic-plastic frames is presented. The formulation of a member tangential stiffness matrix is based on the exact differential equation of a member subjected to large deflection referenced to a local Eulerian coordinate system. Nonli- 


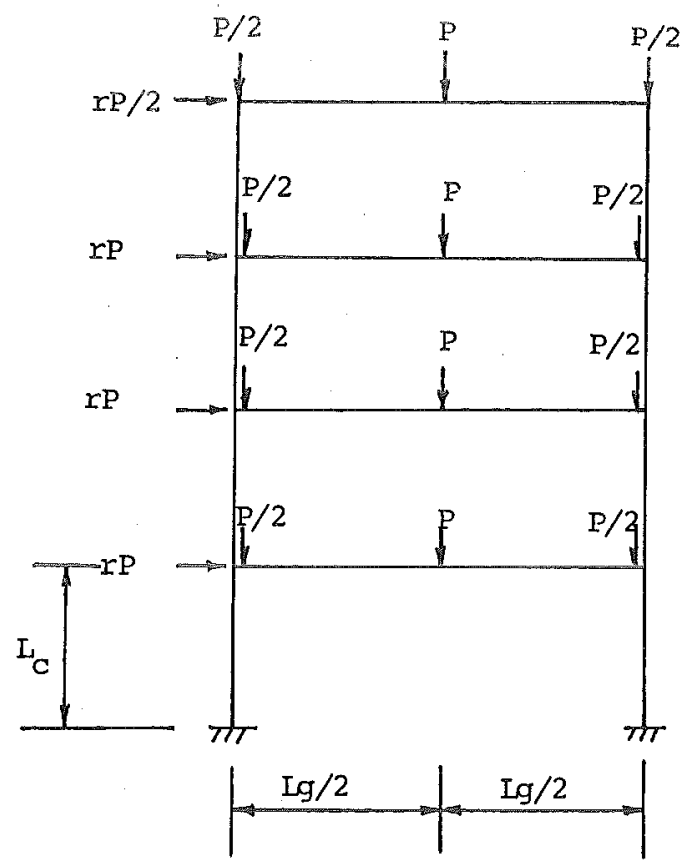

$$
\begin{aligned}
& \text { Columns: W12x79 (Bottom Story) } \\
& \text { W10x60 (Other Stories) } \\
& \text { Girders : } \quad \text { } 16 \times 40 \\
& \mathrm{~L}_{\mathrm{C}}=12 \mathrm{ft}(3.66 \mathrm{~m}) \\
& \mathrm{L}_{\mathrm{g}}=30 \mathrm{ft}(9.15 \mathrm{~m}) \\
& \mathrm{E}=13 \times 10^{3} \mathrm{tsi}\left(201 \times 10^{6} \mathrm{kpa}\right) \\
& \sigma_{0}=15.25 \mathrm{tsi}\left(236 \times 10^{3} \mathrm{kpa}\right)
\end{aligned}
$$

FIG. 9. Four Story Frame = Loads, Dimensions, and Properties

nearities associated with the changes in the stiffness of the member due to axial force, axial shortening of the member, bowing effect, $P$ - $\Delta$ effect, the reduction of plastic moment due to axial loads, and the spreading of plastic zones are included. A $6 \times 6$ stiffness matrix in a global coordinate system is derived for wide-flange members, and its application to the large deffection analysis of inelastic frames is demonstrated by several simple examples. A simpler form of the member tangent stiffness can be obtained if the assumption of small relative member deflection is used. A comparison on the accuracy between the two formulations is also made by the presented examples. It is shown that, for some inelastic frames, if the large displacements are mainly due to the rigid body displacements of members, 


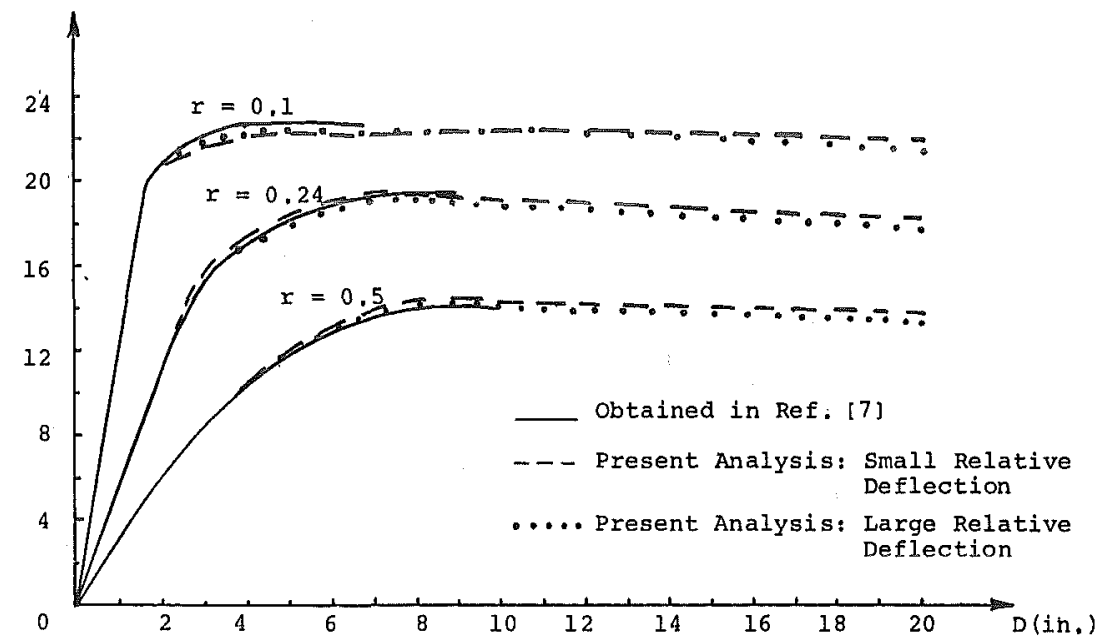

FIG. 10. Load-Displacement Curves for Four-Story Frame (1 ton $=9.97 \mathrm{KN} ; 1$ in. $=$ $25.4 \mathrm{~mm}$ )

the adoption of the small member relative defiection assumption is acceptable and accurate ultimate loads can be obtained.

\section{Appendix I. References}

Alvarez, R. J., and Birnstiel, C. (1969), "Inelastic analysis of multistory multibay frames." J. Struct. Engrg., ASCE, 95(ST11).

Argyris, J. H., Boni, B., Hindenlang, W., and Kleiber, M. (1982). "Finite element analysis of two- and three-dimensional elasto-plastic frames-The natural approach." Comput. Meth. Appl. Mech. Engrg. 35, 221-248.

Backlund, J. (1976). "Large deflection analysis of elastic-plastic beams and frames." Int. J. Mech. Sci. 18, 269-277.

Bathe, J. K., and Ozdemir, H. (1975). "Elastic-plastic large deformation, static and dynamic analysis." Comput. Structures, 6, 81-92.

Cichon, C. (1984). "Large displacements in-plane analysis of elastic-plastic frames." Comput. Structures, 19(5/6), 737-745.

Crisfield, M. A. (1983). "An arc-length method including line searches and accelerations." Int. J. Num. Meth. Engrg., 19, 1269-1289.

El-Zanaty, M. H., and Murray, D. W. (1983). "Nonlinear finite element analysis of steel frames." J. Struct. Engrg., ASCE, 109(2), 353-368.

Harung, H. S., and Miller, M. A. (1973). "General failure analysis of skeletal plane frames." J. Struct. Engrg., ASCE, 99(ST6).

Hauck, G. F., and Lee, S. L. (1963). "Stability of elasto-plastic wide-flange columns." J. Struct. Engrg., ASCE, 89(ST6).

Kam, T. Y., Rossow, E. C., and Corotis, R. B. (1983). "Inelastic tangential stiffness for 2-D frames." J. Struct. Engrg., ASCE, 109(11), 2685-2697.

Kassimali, A. (1983). "Large deformation analysis of elastic-plastic frames." $J$. Struct. Engrg., ASCE, 109(8), 1869-1886.

Kuo, C. C. (1985). "Large deflection analysis of elastic-plastic frames," thesis presented to the National Chiao Tung University, at Hsinchu, Taiwan, in partial fulfillment of the requirements for the degree of Master of Engineering. 
Lee, S. L., Manuel, F. S., and Rossow, E. C. (1968), "Large deflections and stability of elastic frames." J. Engrg. Mech. ASCE, 94(EM2), 521-547.

Meek, J. L., and Tan, H. S. (1984). "Geometrically nonlinear analysis of space frames by an incremental interactive technique." Comput. Meth. Appl. Mech. Engrg., 47, 261-282.

Oran, C. (1973). "Tangent stiffness in plane frames." J. Struct. Engrg., ASCE, 99(ST6), 973-985.

Oran, C., and Kassimali, A. (1976), "Large deformations of framed structures under static and dynamic loads." Int. J. Comput. Struct., 6, 539-547.

Qashu, R. K., and Dadeppo, D. A. (1983). "Large deflection and stability of rigid frames." J. Engrg. Mech, ASCE, 109(3), 765-780.

Saafan, S. A. (1963). "Nonlinear behavior of structural plane frames." J. Struct. Engrg., ASCE, 89(ST4), 557-579.

Schilling, C. G., Schutz, F. W., and Beedle, L. S. (1956). "Behavior of welded single-span frames under combined loading." Welding J. Res. Suppl. 35.

Timoshenko, S. P., and Gere, J. M. (1961). Theory of elastic stability, 2nd ed. McGraw-Hill Book Co., Inc., New York, NY.

Turner, M. J. et al. (1960). "Large deflections of structures subjected to heating and external loads." J. Aerospace Sci., 27(2), 97-106.

Vijakklane, C., Nishino, F,, and Lee, S. L. (1974). "Inelastic stability of unbraced building frames." J. Struct. Engrg., ASCE, 100(ST3), 645-666.

\section{Appendix II. Notation}

The following symbols are used in this paper:

$$
\begin{aligned}
a & =\left[\begin{array}{ll}
\dot{a}_{1} & a_{2}
\end{array}\right] \\
a_{1} & =\left[P M_{i} M_{j} y_{1} \ldots y_{n-1} r_{1} \ldots r_{n-1}\right] \\
a_{2} & =\left[\begin{array}{ll}
u & y_{i}^{\prime}
\end{array}\right] \\
B & =\text { displacement transformation matrix; } \\
b & =\left[P M_{i} M_{j}\right]^{t} ; \\
\mathbf{F} & =\text { displacement equation vector; } \\
F_{i} & =\text { end displacement equation; } \\
H_{k} & =\text { equations of intermediate vertical displacements; } \\
K_{k} & =\text { equation of intermediate slopes; } \\
K & =6 \times 6 \text { stiffness matrix; } \\
K^{\prime} & =3 \times 3 \text { stiffness matrix; } \\
L & =\text { length of deformed member; } \\
L_{0} & =\text { initial length of member; } \\
M_{j} & =\text { end moments; } \\
P & =\text { axial force along local coordinate axis; } \\
q & =\left[y_{1} \ldots \ldots \ldots \text {. } y_{n-1} r_{1} \ldots \ldots r_{n-1}\right] ; \\
T & =\text { force transformation matrix; } \\
u & =\text { axial displacement; } \\
\beta & =\text { angle between local and global coordinate axes; and } \\
\theta_{i}, \theta_{j} & =\text { end rotations. }
\end{aligned}
$$

